Available online on 25.06.2019 at http://ajprd.com
(c) 2013-19, publisher and licensee AJPRD, This is an Open Access article which permits unrestricted non-
commercial use, provided the original work is properly cited

Open ${ }^{\circ}$ Access

Review Article

\title{
MAGNETIC AND pH SENSITIVE NANOPARTICLES FOR CANCER DRUG DELIVERY
}

\author{
Jadhao Amol G*1, Bhosale Akashy G², Sitaphale G $\mathbf{R}^{3}$, Rajguru Jeevan $\mathbf{R}^{1}$, Nagare \\ Sonali A ${ }^{3}$, Deshmane S. V.3 \\ ${ }^{1}$ Master of Pharmacy in Pharmaceutics at Anuradha College of Pharmacy, Chikhli Dist-Buldana M.S. \\ India \\ ${ }^{2}$ Master of Pharmacy in Quality assurance at Anuradha College of Pharmacy, Chikhli Dist- Buldana \\ M.S. India \\ ${ }^{3}$ Department of Pharmacognosy, Anuradha College of Pharmacy, Chikhli Dist- Buldana M.S. India
}

\begin{abstract}
A B S T R A C T
Cancer remains a leading cause of death worldwide with more than 10 million new cases every year. Tumor-targeted nanomedicines have shown substantial improvements of the therapeutic index of anticancer agents, addressing the deficiencies of conventional chemotherapy, and have had a tremendous growth over past several decades. Due to the pathophysiological characteristics that almost all tumor tissues have lower $\mathrm{pH}$ in comparison to normal healthy tissues, among various tumortargeted nanomaterials, pH-responsive polymeric materials have been one of the most prevalent approaches for cancer diagnosis and treatment. In this review, we summarized the types of $\mathrm{pH}$-responsive polymers, describing their chemical structures and $\mathrm{pH}$ response mechanisms; we illustrated the structure-property relationships of pH-responsive polymers and introduced the approaches to regulating their $\mathrm{pH}$-responsive behaviors; we also highlighted the most representative applications of $\mathrm{pH}$-responsive polymers in cancer imaging and therapy. Many strategies based on stimuli-responsive nanocarriers have been developed to control the drug release and avoid premature release. Here, we focus on the use of the subtle changes of $\mathrm{pH}$ between healthy and diseased areas along the body to trigger the release of the cargo. The application of nanotechnology to medicine constitutes a major field of research nowadays. In particular, the use of mesoporous silica and carbon nanoparticles has attracted the attention of numerous researchers due to their unique properties, especially when applied to cancer treatment.
\end{abstract}

Keywords: $\mathrm{pH}$ responsive polymers; nanomedicine; Mesoporous nanoparticles, tumor imaging; drug delivery

\begin{tabular}{l}
\hline A R T I C L E I N F O: Received 12 May 2019; Review Completed 03 June 2019; Accepted 22 July 2019; Available online 15 August 2019 \\
Cite this article as: \\
Amol G. Jadhao, Akashy G. Bhosale2, G. R Sitaphale3, Jeevan R. Rajguru4, Sonali A. Nagare5 S. V. Deshmane, Magnetic \\
and Ph Sensitive Nanoparticles for Cancer Drug Deliverys Asian Journal of Pharmaceutical Research and Development. \\
2019; 7(4):60-71, DOI: http://dx.doi.org/10.22270/ajprd.v7i4.540
\end{tabular}

\section{INTRODUCTION}

$\mathrm{T}$ The application of nanotechnology in health and medicine, so-called nanomedicine, is one of the most promising and exciting research areas nowadays.
The design of nanoparticles to address disease and to monitor and protect human health is expected to revolutionize the medical field in the next few decades. In this sense, the capacity of producing nanoparticles in the range of 20-200 $\mathrm{nm}$ in diameter has fueled the design of 
materials large enough to escape clearance through the kidney and small enough to present long circulation times into the bloodstream. Although nanoparticles have been applied against lung, kidney, rheumatoid arthritis, diabetes or neurodegenerative diseases ${ }^{1-5}$, among others, the first steps of nanomedicine research have been mainly focused on the challenging area of cancer, either on diagnosis or treatment. Current cancer treatments concern the use of radiotherapy, surgery or chemotherapy. Unfortunately, chemotherapeutics are administered systemically and do not show selectivity for cancer cells. Because of that, chemotherapy is considered to be aggressive for patients, since the drugs distribute all along the body instead of only near the damaged area. Then, it would be desirable to target the chemotherapeutics only to cancer cells. Nanotechnology is a rapidly expanding field, encompassing the development of man-made materials in the 5-200 nanometer size range. This dimension vastly exceeds that of standard organic molecules, but its lower range approaches that of many proteins and biological macromolecules. In the scientific world, the term "nano" is, however, somewhat ambiguous since it does not designate the same reality for physicists, chemists and biologists. Conceptually, nanotechnologies in general and nanoparticles in particular have revolutionized the administration of medicines. An ideal nanoparticle for drug delivery should be able to entrap the maximum amount possible of pharmaceutical agents. To avoid that, the surface of the nanoparticles can be decorated with hydrophilic layers to prevent opsonization (and therefore, the action of macrophages) ${ }^{6-8}$. The nanoparticles can accumulate in the tumor via passive targeting due to the abnormal vasculature of solid tumors (the so-called Enhanced Permeability and Retention effect) ${ }^{9}$ and then selectively internalize the cancer cells via active targeting

\section{Types of Nanoparticles:}

\section{Non-Porous}

In the last few years, a number of non-porous nanoparticles for biomedical applications have been developed. It is possible to find both organic and inorganic platforms, such as liposomes, polymeric nanoparticles, dendrimers, magnetic nanoparticles or metal nanoparticles, among others $^{11}$. In fact, there are some examples of those nonporous platforms commercially available, such as: (1) paramagnetic iron oxide nanoparticles (e.g., Venofer, Resovist); (2) liposomes (e.g., Doxil, Caelyx); (3) biodegradable polymer nanoparticles (e.g., Somavert, Cimzia); or even (4) drug-antibodies conjugates, in which those antibodies guide the drug towards the targeted cells (e.g., Mylotarg) ${ }^{12}$.

\section{Mesoporous Nanoparticles}

Mesoporous nanoparticles have recently attracted the attention of nanomedicine researchers thanks to their robustness, their high loading capacity and the easy chemical functionalization of their surface. This offers a great potential for targeted drug delivery and controlled release of chemotherapeutic drugs. Mesoporous Silica Nanoparticles. The synthesis of mesoporous silica nanoparticles (MSNs) is based on the sol-gel process, in which successive hydrolysis and condensation steps of the silica precursors lead to the formation of a network of silica. That polymerization process is carried out in the presence of surfactants as structure-directing agents (Figure1a). The final meso structure would depend on the type and concentration of surfactants and also on certain experimental parameters, such as temperature. Then, removing the surfactant would lead to mesoporous materials with empty mesopores, where drug molecules would be loaded. A modification of the Stöber method is employed to obtain nanoparticles with mesoporous structures, which is based on using very dilute conditions during the sol-gel process. In a typical synthesis, cetyl trimethyl ammonium bromide (surfactant) is dissolved in pure water in the presence of sodium hydroxide so that the surfactant organizes in cylindrical micelles. After that, tetraethyl orthosilicate (silica precursor) is added dropwise, and the silica network is formed through the hydrolysis and condensation of the silica precursor around the micelles. Finally, the surfactant is removed from the mesopores through extraction with either a methanol/HCl or ethanol/ammonium nitrate solution, giving rise to nanoparticles with a hexagonal distribution of empty pores (Figure 1b).

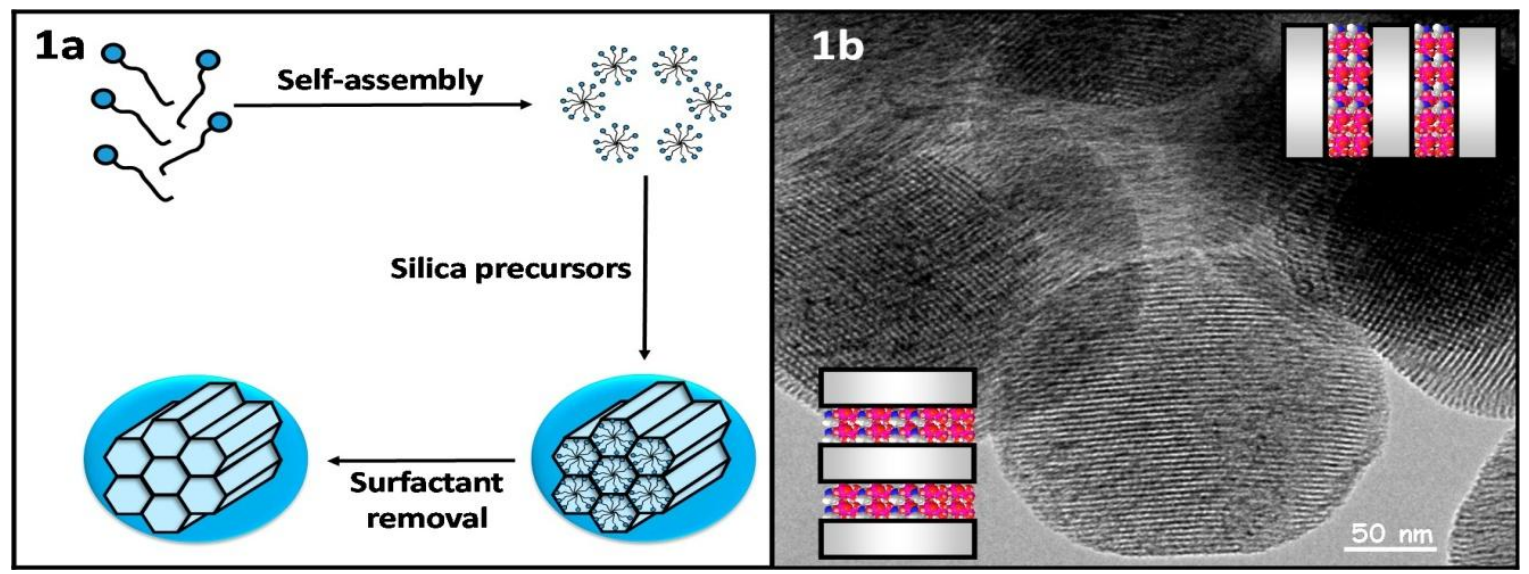

Figure 1: (a) Scheme synthesis of mesoporous silica nanoparticles through a modified Stöber (b) Transmission Electron Microscopy micrography of the pore distribution of MCM-41-type mesoporous silica nanoparticles. 


\section{Mesoporous Carbon Nanoparticles}

In the last few years, mesoporous carbon nanoparticles have received increasing attention by nanomedicine researchers since mesoporous carbon nanoparticles (MCNs) show similar structural properties as MSNs. Moreover, MCNs show enhanced laser absorption in the near-infrared (NIR) region that leads to an efficient conversion of light into heat that can be applied to antitumor therapy ${ }^{13}$. The synthesis of MCNs can be accomplished by either hard or soft templating approaches ${ }^{14}$. The hard templating approach is based on the use of presynthesized organic or inorganic templates. The template serves as a mold for the replication of the mesoporous carbon materials, and no significant interactions between the carbon precursors and the template are observed. A typical synthesis involves four steps: (1) preparation of the porous silica template; (2) impregnation of the silica template with the precursors; (3) cross-linking and carbonization of the organic precursors (e.g., phenolic resins, sucrose); and (4) dissolution of the silica template. On the other hand, the soft templating approach is based on the generation of nanostructures through the self-assembly of organic molecules, such as surfactants.

\section{Stimuli-Responsive Nanoparticles}

As has been mentioned before, nanoparticles are attractive in drug delivery applications due to their ability to host drugs within them. An efficient drug delivery system would travel along the body without releasing the cargo until reaching the diseased area, where the drugs would be released. To achieve that, the concept of stimuli-responsive drug delivery arises. It is based on the use of stimuli to trigger the release of the payload only under specific conditions, i.e., those of the damaged area. This concept is particularly useful when using mesoporous silica or carbonbased nanoparticles because they present an open structure. Then, the drugs can be loaded through diffusion within them, but the cargo can also diffuse out when placed in aqueous media. This clashes with the desired release of the drugs only in the target area. Although the electrostatic interactions between nanocarriers and guest molecules may help to retain the cargo, as will be seen in the following section, an extended strategy is the use of molecular structures capable of blocking the mesopores. These structures are referred to as gatekeepers, which are organic or inorganic structures capable of hampering the release at physiological conditions and suffering some kind of structural change when a certain stimulus is applied, which would open the pores and trigger the drug release. The gates can be opened through external or internal stimuli (Figure 2).

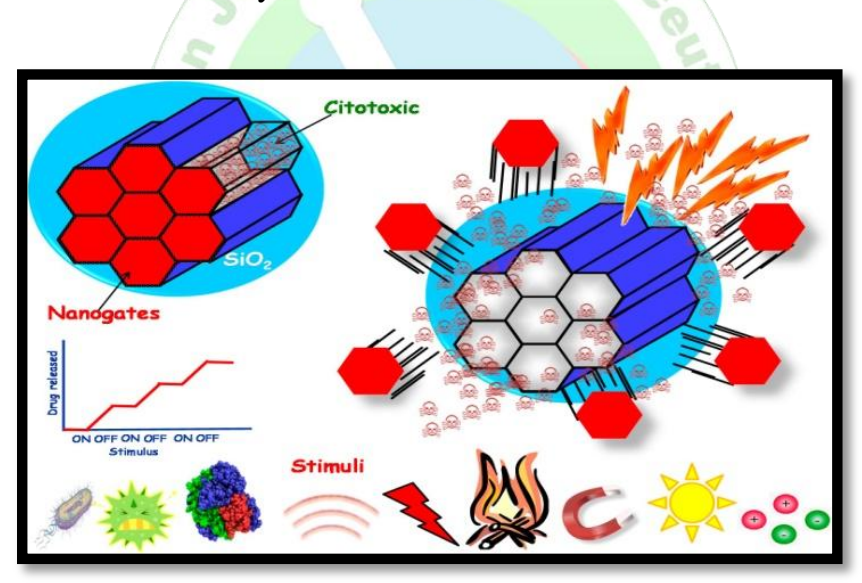

Figure 2: Stimuli-responsive nanoparticles where the drug release can be triggered through the application of many different stimuli.

\section{External Stimuli-Responsive Nanocarriers}

External stimuli-responsive nanocarriers are those in which the opening of the pores is mediated by some stimulus coming from the outside of the body. The main advantage of this kind of system is that the stimulus can be modulated, opening and closing the gates on-demand in many cases, as they are applied through external equipment. The literature provides examples of temperature ${ }^{15-16}$, magnetic ${ }^{17-18}$, light ${ }^{19-21}$ or ultrasound-sensitive ${ }^{22}$ systems. The use of magnetic and thermo-responsive nanoparticles has also been reported, as they are synergic stimuli because heat can be generated by applying an alternating magnetic field ${ }^{23-24}$.

\section{Internal Stimuli-Responsive Nanocarriers}

Internal stimuli-responsive nanocarriers are those in which the behavior of the gate is modulated by some stimulus from the inside of the body. It is well-known that during some diseases, the determined enzymes may be overexpressed $^{25}$ or the concentration of reducing agents may increase inside the cell with respect to the outside ${ }^{26}$. These abnormal situations lead to enzyme- ${ }^{27-28}$ and redoxresponsive ${ }^{29-30}$ drug delivery systems, among others. Interestingly, as will be discussed during the following sections, the value of the $\mathrm{pH}$ is not kept constant along the body. Then, these subtle changes in $\mathrm{pH}$ can be taken advantage of to design $\mathrm{pH}$-responsive drug delivery systems that only release the payload when the $\mathrm{pH}$ deviates from the physiological value.

\section{The Role of pH in Drug Delivery}

The maintenance of the different physiological pHs along the body is of vital importance for the organism to keep alive. For instance, variations in $\mathrm{pH}$ of body fluids within 7.35 and 7.45 can be corrected, but greater alterations may lead to acidosis or alkalosis events that may even cause 
death. However, that rule does not always apply, and these variations can be taken advantage of in different scenarios throughout the body to design $\mathrm{pH}$-responsive nanocarriers.

\section{Host-Guest Interactions}

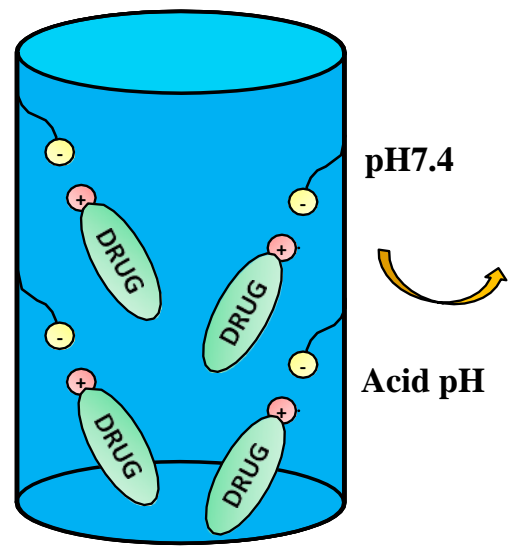

An extended strategy to provide the nanocarriers with $\mathrm{pH}$ sensitivity is through the electrostatic interaction between the functional groups from the carrier and those of the drugs at physiological $\mathrm{pH}$, as well as the lack of them when $\mathrm{pH}$ varies (Figure 3 ).

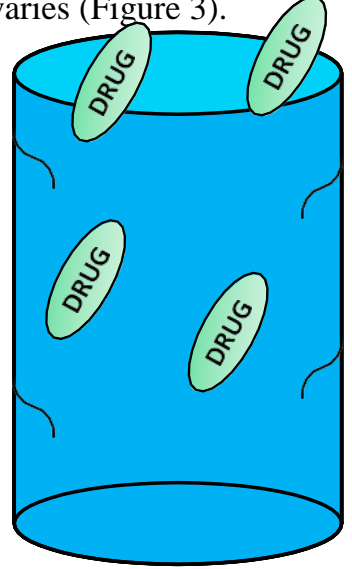

Figure 3: Example of host-guest interaction-based drug delivery involving cationic drugs.

Depending on the ionic nature of the therapeutic agents, a specific nanocarrier functionalization should be chosen. Generally speaking, positively-charged nanocarriers should be used when using an anionic drug in order to maximize the loading capacity and achieve a controlled released and vice versa ${ }^{32-34}$. One of the most used drugs against cancer is doxorubicin (DOX). DOX is a cationic drug that has been widely used for more than 30 years to treat different types of tumors. It has shown great treatment potential, but its lack of cancer cell selectivity makes it a double-edged sword because it kills not only malignant, but also healthy cells. The action mechanism is complex, but, briefly, it enters the cell through diffusion due to its high affinity to bind to the proteasome of the cytoplasm. Once there, it interferes with the topoisomerase II-DNA complex, leading to the formation of double-stranded breaks of the DNA, causing cell death ${ }^{35}$. Although the literature on mesoporous nanoparticles mostly provides DOX-based papers, the study of the host-guest interactions with some other drugs, such as ibuprofen, 5-fluoracil, mitoxantrone, methotrexate or sulfasalazine, has also been reported ${ }^{31}$.

\section{2.pH-Responsive Polymers Fabricating}

$\mathrm{pH}$ responsive polymeric nanomaterials has been rapidly developed after the emerging of advanced techniques in polymer synthesis, especially reversible-deactivation radical polymerization such as ATRP and RAFT ${ }^{36-37}$ The technical state of the art allows for precise control over molecular weights and molecular weight distributions, affording polymers with well-defined topology structure ${ }^{38-40}$.

The $\mathrm{pH}$ responsive polymers can be mainly classified into two categories:

\section{Polymers with ionisable moieties}

\section{Polymers that contain acid-labile linkages}

\section{pH Responsive Polymers with Ionizable Groups}

The $\mathrm{pH}$ response arises from the reversible protonation and deprotonation of ionizable groups at the molecular level.
The $\mathrm{pKa}$ of a polyelectrolyte, which is defined as the $\mathrm{pH}$ with equal concentration of the protonated and deprotonated forms, can serve as a critical benchmark to reflect the polymer ionization behaviors at various $\mathrm{pH}$ levels ${ }^{41}$. In general, there are two types of ionizable polymers: basic polymers that accept protons at a relatively low $\mathrm{pH}$ and acidic polymers that release protons at a relatively high $\mathrm{pH}$. Consequently, these polybases or polyacids can form positively or negatively charged polymer chains at different pH levels. Common basic moieties include amines, pyridines, morpholines, piperazines; and common acidic groups include carboxylic acids, sulfonic acids, phosphoric acids, boronic acids, etc. ${ }^{30}$. Among them, amines, especially tertiary amines, have drawn particular attention due to their ease to prepare and the feasibility to finely tune their $\mathrm{pKa}^{42-}$ 43. It was reported that the amines can present a marginally lower $\mathrm{pKa}$ when substituted with longer hydrophobic chains ${ }^{44}$. Recently, designed a series of ultra-pH sensitive (UPS) polymers for real-time tumor imaging ${ }^{45-48}$. These nanoprobes can sharply respond to and amplify in vivo $\mathrm{pH}$ signals in a very narrow $\mathrm{pH}$ span. The UPS nanoparticles are comprised of an amphiphilic block copolymer: PEG-bPMA, where PEG stands for hydrophilic poly (ethylene glycol) and PMA is a hydrophobic segment based on poly methacrylates with tertiary amine substituent. At physiological pH (7.4), the block copolymers stay as coreshell micelles driven by self-assembly. Acid-triggered dissociation of micelles enable the increase of fluorescence intensity for molecular imaging and release of cargo for drug delivery. Yan et al. further expand the UPS polymer design based on biodegradable polypeptides (Figure 4) 49. They modified the peptides with ionizable tertiary amines for $\mathrm{pH}$ responsive behavior. Hydrophilic and hydrophobic blocks were synthesized independently and covalently connected by the copper (I)-catalysed alkyne-azide cyclo addition (CuAAC) "click" chemistry ${ }^{50}$. The copolymer's $\mathrm{pKa}$ can be readily tuned by altering the ratio of amino substituents. Similar to aforementioned UPS designing, a fluorescent cyanine dye (Cy5.5) was attached at the chain end of the amphiphilic copolymer to convert the subtle $\mathrm{pH}$ 
variation into significant fluorescence intensity change. This study suggested that the ultra-pH sensitivity resulted from the reversible protonation of ionizable amines rather than the peptide backbone. It also in spired the design of degradable ultra-pH sensitive polymers.

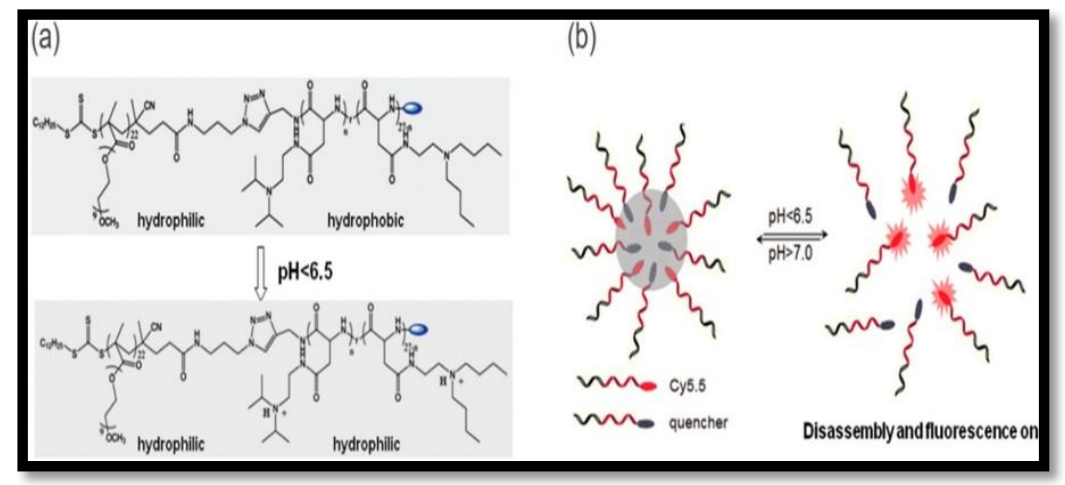

Figure 4: Design of ultra-pH-sensitive polypeptide micelles. (a) Structure of amphiphilic copolymers with ionizable tertiary amines and their transition at lower $\mathrm{pH}$. (b) $\mathrm{pH}$-triggered disassembly and fluorescence.

Natural macromolecules with ionizable amino acid residues have also been investigated for $\mathrm{pH}$ triggered delivery of imaging and therapeutic agents ${ }^{51-55}$. Engel man and colleagues have been working on the development of novel pH-responsive transmembrane peptides, $\mathrm{pH}$ low insertion peptide ( $\mathrm{pH}$ LIPs), for basic research and translational applications in membrane biophysics and medicine (Figure $5)^{56}$. These $\mathrm{pH}$-responsive peptides were derived from the C-helix of the protein bacterio rhodopsin ${ }^{57}$. pH LIPs spontaneously self-assemble into a helix structure and insert across the membrane upon exposure to acidic environment ${ }^{58}$. In physiological condition, where the $\mathrm{pH}$ is around 7.4, the ionizable acid residues of the pHLIP (red circles) stay negatively charged and the peptide will be weakly bound to the surface of membrane. Once encountering acidic condition like tumor microenvironment, carboxyl group will be protonated and neutralized. Increased lipophilicity (green circles) as a result of ionization drastically enhances the affinity of $\mathrm{pH}$ LIP to the hydrophobic inner core of the cellular membrane and triggers the formation of a helix and ensuing insertion across the membrane. When the protonatable carboxyl groups are exposed to the normal intracellular $\mathrm{pH}$, pHLIP gets reversibly deprotonated and anchors in the membrane. The pH-responsive behavior of pHLIP can be easily modified by replacing ionizable aspartic acid residues with glutamic acid residues ${ }^{60-61}$ or positively-charged lysine residues ${ }^{59}$, or other protonatable amino acids ${ }^{62}$.

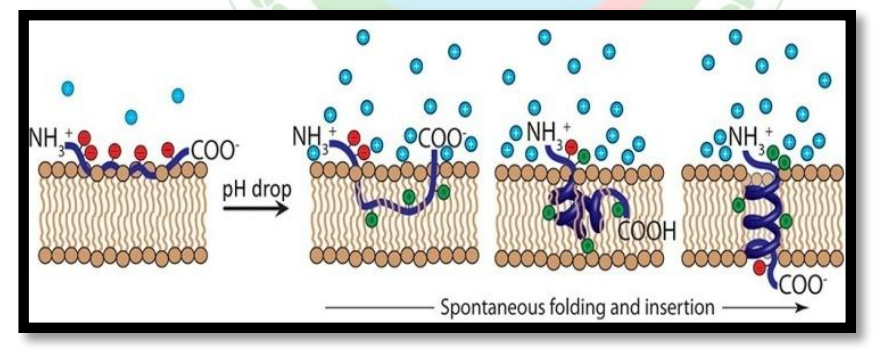

Figure 5: Mechanism of $\mathrm{pH}$ (Low) Insertion Peptide (pHLIP)

Insertion into the Cellular Membrane. In healthy tissue where the $\mathrm{pH}$ is around 7.4, the ionizable residues of the pHLIP (red circles) remain deprotonated and negatively charged, and the peptide resides at or near the hydrophilic surface of the cellular membrane. Upon exposure to acidic tumor microenvironment, the ionizable residues and negatively charged C-terminal carboxyl group of the pHLIP become neutrally charged (green circles). The protonation results in an increase in the hydrophobicity of the pHLIP, triggering the pHLIP to spontaneously fold into a helix and insert across the hydrophobic lipid bilayer of cell membrane, resulting in the formation of a transmembrane helix. Following internalization and exposure to cytosol with $\mathrm{pH}$ above 7, C-terminal ionizable residues are deprotonated again and anchor the pHLIP into the membrane.
Polyacids have also been extensively investigated for the design of $\mathrm{pH}$-responsive nanoplatforms. Hyaluronic acid (HA) is a key component of the extracellular matrix and is known to bind to CD44 proteins as a surface receptor on cancer cells ${ }^{63}$. Kono and coworkers reported HAbased $\mathrm{pH}$ sensitive polymer-modified liposomes for tumor-targeted delivery of chemotherapeutics ${ }^{64}$. Instead of simply using HA as targeting moiety, these authors introduced 3-methyl glutarylated (MGlu) units and 2-carboxycyclohexane-1carboxylated (CHex) units for the design of a new class of pH-responsive polymers with transition $\mathrm{pH}$ around 5.4-6.7. Carboxyl group-introduced HA derivatives were prepared by reaction with various dicarboxylic anhydrides. Terminal alkyl chains served as anchor units inserting into the hydrophobic lipid bilayers of liposomes (Figure 6). 


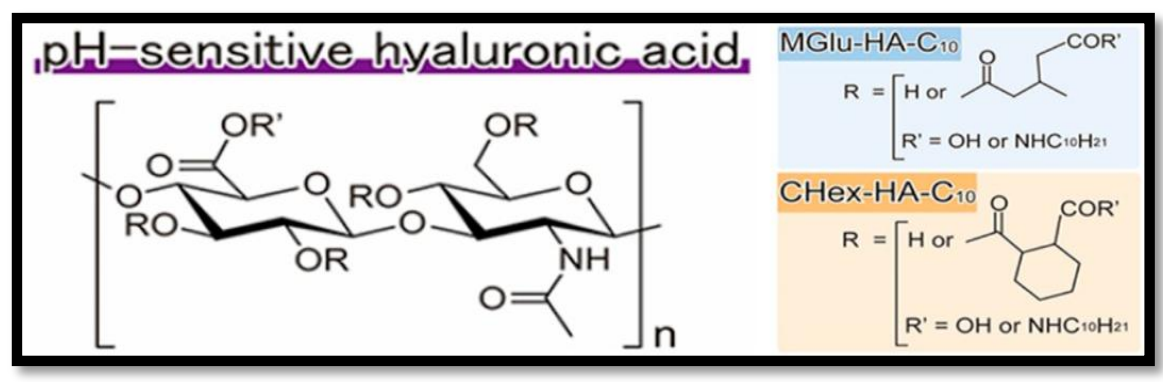

Figure 6: Structures of $\mathrm{pH}$-sensitive modified hyaluronic acid derivatives.

\section{pH Responsive Dissociation Based on Acid-Labile Linkages}

Polymers containing acid-labile or base-labile linkages can respond to the change of $\mathrm{pH}$ by degradation. Since the tumor microenvironment is slightly acidic compared to normal physiological environment, polymeric nanoparticles containing base-labile linkages have seldom be enused for cancer therapy and will not be covered in the perspective. In contrast, polymers with acid-labile linkages have been extensively utilized for the designing of anticancer drug delivery systems ${ }^{65}$.

Hydrazone is one of the most explored acid-labile linkages used for acid-responsive dissociation release due to its easy synthesis and moderate sensitivity ${ }^{66}$. Incorporation of hydrazine into the backbone of polymers represents an ideal strategy for the design of tumor-targeted drug delivery systems. Nie group designed a pH-sensitive drug-gold nanoparticle system for tumor chemotherapy and surface enhanced Raman scattering (SERS) imaging (Figure 7) ${ }^{67}$. This multifunctional system comprised of poly (ethylene glycol), doxorubicin (Dox), hydrazone linker, and gold nanoparticles (Au-Dox-PEG). 3-[2-pyridyldithio] propionyl hydrazide (PDPH) was conjugated with Dox in methanol. PDPH acted as a $\mathrm{pH}$-sensitive linker and introduced thiol groups to ensure the anchoring of drug conjugates onto the surface of gold nanoparticles. Gao and coworkers developed a $\mathrm{pH}$ responsive polypeptide-drug nanoparticles for targeted cancer therapy based on welldefined elastin-like polypeptides and acid-labile hydrazone linker ${ }^{68}$. Wang reported a hydrazone-based multifunctional sericin nanoparticles for $\mathrm{pH}$-sensitive subcellular delivery of anti-tumor chemotherapeutics ${ }^{69}$

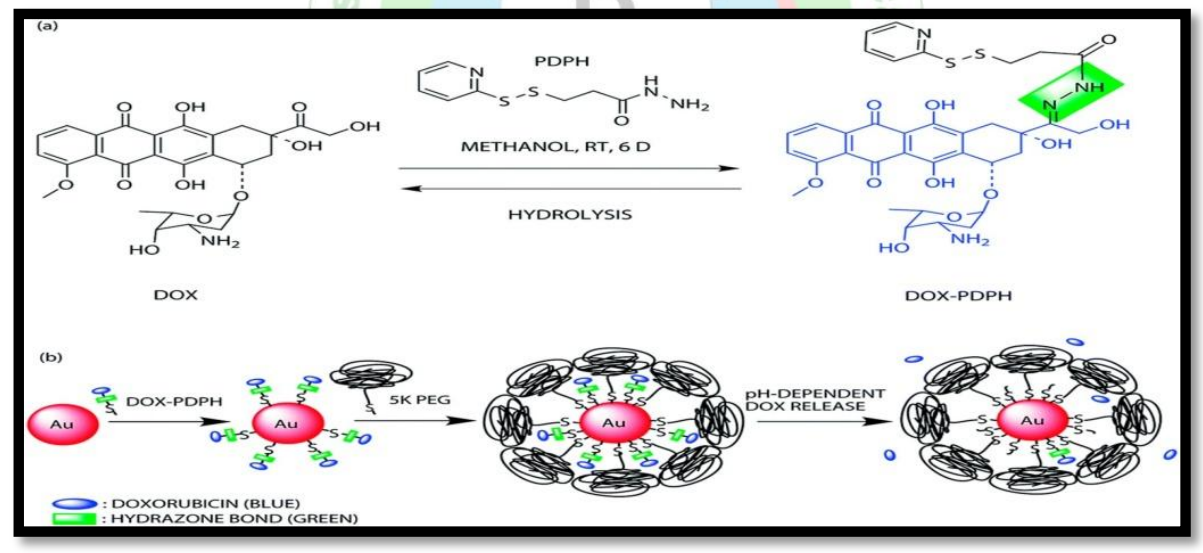

Figure 7: A pH-sensitive drug-gold nanoparticle system. (a) Chemical synthesis of the doxorubicinhydrazone linker conjugates (dox-PDPH). (b) Schematic illustration for the synthesis of the multifunctional drug delivery system and its $\mathrm{pH}$-dependent doxorubicin release.

Acetal and ketal are also commonly used acid-labile linkages, which are very stable under basic conditions, but can be readily hydrolyzed to corresponding carbonyl compound (aldehyde and ketone) and alcohols through a caboxonium ion intermediate upon acidic cleavage ${ }^{70} . \mathrm{Lu}$ and coworkers reported a novel envelop-like mesoporous silica nanoparticle platform ${ }^{71}$. This system immobilized acetals on the surface of silica (yellow, left) before coupling to gate-keeper nanoparticle (purple, right) (Figure 8). At acidic $\mathrm{pH}$, the acetal was effectively hydrolyzed to remove the gate keepers, allowing the escape of entrapped drug molecules. Liu et al. reported the facile fabrication of acidsensitive polymersomes for intra cellular release of drug over several days ${ }^{72}$. The polymersomes compromising cyclic benzylidene acetals in the hydrophobic bilayers were stable under neutral $\mathrm{pH}$, whereas were hydrolyzed into hydrophilic diol moieties upon exposure to acidic $\mathrm{pH}$ milieu. The pH-triggered hydrolysis can be easily monitored by many experimental methods including UV/Vis spectroscopy and TEM. A novel class of acid degradable poly (acetal urethane) was also reported for the construction of acid-degradable micelles for delivery of hydrophobic anti-tumor therapeutics ${ }^{73}$. More recently, Wang group designed a new hyper branched amphiphilic acetal polymers for $\mathrm{pH}$-sensitive drug delivery ${ }^{74}$. Under neutral conditions, the block copolymers self-assembled into well-defined core-shell micelles. This $\mathrm{pH}$-induced acetal cleavage resulted in the drastic decrease of hydrophobicity and dissociation of micelles. De Geest and coworkers synthesized ketal-containing block copolymers 
as pH-responsive nanocarriers for the hydrophobic anticancer drug paclitaxel (PTX) 75. The hydrolysis of the ketal groups in the block copolymer side chains at $\mathrm{pH}<5$ lead to decomposition of the block copolymer nanoparticles and the PTX release.

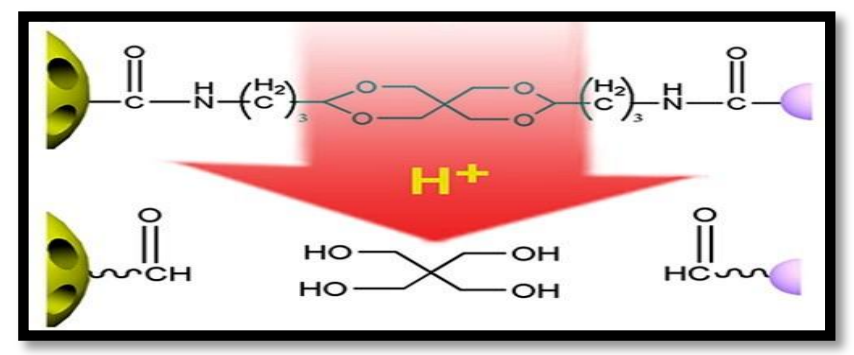

Figure 8: Schematic illustration of the envelope-type mesoporous silica nanoparticle for $\mathrm{pH}$ responsive drug delivery.

Boronate esters, formed between a boronic acid and an alcohol, are stable at neutral or alkaline $\mathrm{pH}$ and readily dissociate to boronic acid and alcohol groups in a low-pH environment. Boronate esters have been widely employed to exploit $\mathrm{pH}$-sensitive polymeric carriers for anticancer drug delivery. Messersmith et al. conjugated the boronic acid containing anticancer drug bortezomib (BTZ) to catechol-containing polymers via the boronate ester (Figure 9) [76]. Under neutral or basic condition, BTZ and catechol formed stable conjugates via boronate ester linkers, deactivating the cytotoxicity of BTZ. Upon exposure to

Figure 9: Catechol-containing polymers conjugated the boronic acid containing anticancer drug bortezomib via acid-labile boronate esters. Boronate esters are

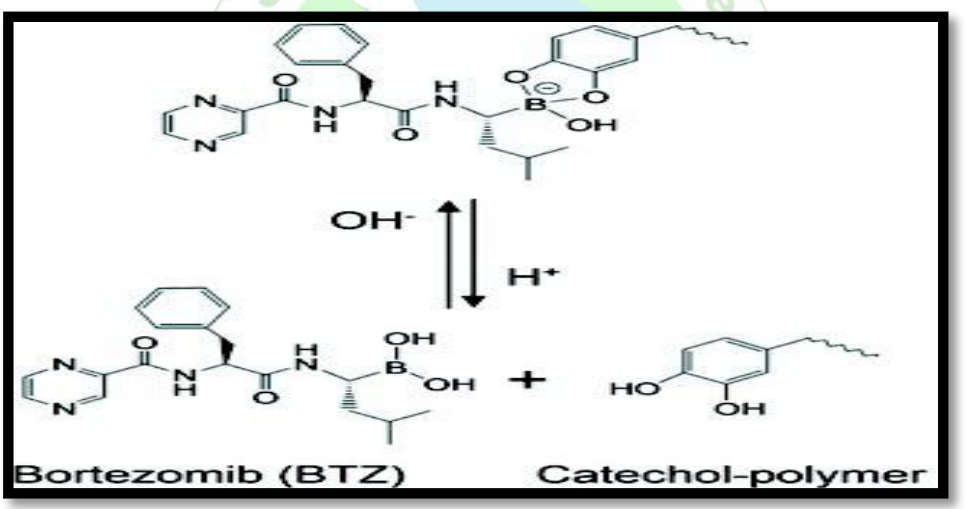

stable at neutral or alkaline $\mathrm{pH}$ and readily dissociate to boronic acid and alcohol groups in acidic environments to release the free active drug.

\section{Synthesis methods of magnetic nanoparticles}

Methods based on preparation from solution allow the formation of magnetic NPs with precise controlled size and shape in a simple manner.

\section{Homogeneous precipitation}

Homogeneous precipitation methods are used in order to achieve uniform particles. These methods have as basis the separation of the nucleation and growth of the nuclei. When the concentration of continuant species attains critical supersaturation, one short burst of nucleation appears and so the nuclei are synthesized. They can grow consistently by means of diffusion of the solutes from the solution on their surface, up to when they achieve their final size. These two phases must be distinct in order to attain monodispersity, meaning that once the growth process is established, nucleation must be avoided. However, uniform shaped nanoparticles have also been realized after numerous lower $\mathrm{pH}$, the conjugates readily release free drugs. Levkin and coworkers reported a dextran-based $\mathrm{pH}$-sensitive nanoparticle system by modifying vicinal diol of dextran with boronate esters. PH-triggered hydrolysis of ester linkers resumed the hydrophilic hydroxyl groups of dextran and destabilized the dug-encapsulated nanoparticles. Kim et al. reported a $\mathrm{pH}$-sensitive nanocomplex bygrafting phenylboronic acid (PBA) onto the backbone of poly (maleic anhydride). Dox and PBA readily formed boronate esters following simple mixing. 
However, the downside is that the particle size distribution cannot be controlled ${ }^{77-78}$.

\section{Microemulsion methods}

Water-in-oil (w/o) microemulsions are liquid solutions with properties as thermodynamic stability, isotropy, transparence and are also simple and versatile starting materials which can furnish nanosized particles. This type of systems consists of a continuous oil phase, in which dispersed assemblies of surfactant molecules can be found. In these assemblies of surfactant molecules, there are entrapped micro droplets of the aqueous phase. The limitation of particle nucleation, growth and cluster formation is given by the surfactant-stabilized microcavities. There have been conducted studies using this synthesis method, the result being magnetic NPs with an average size of $4 \mathrm{~nm}$ up to $12 \mathrm{~nm}$ and a standard deviation of 0.2 up to 0.3 . These types of emulsions are used to synthesize super paramagnetic iron oxide nanoparticles with controlled size and shape. The main asset of this method is the controlled size of the NPs that can be obtained by managing the dimension of the aqueous droplets core.

\section{Polyol method}

Polyol solvents, such as PEG, have unique properties: from the dielectric point of view, they behave as solvents capable to dissolve inorganic compounds; from their high boiling point of view, they present a large operating temperature range in the concern of synthesis of inorganic compounds. The polyol liquid is the solvent of the metallic precursor and the reducing agent. The metallic precursor can be either very soluble or lightly soluble in the polyol. The solution is then mixed up and heated to a given temperature. Nonaggregated and well-defined particles can be obtained by managing the kinetics of reactions in solution. The polyol method was the first technique that used noble metals (e.g., gold) and nickel, cobalt or copper. Recently, the method has widespread and was used to synthesize other materials, as iron based alloys. Non aggregated magnetite nanoparticles can be synthesized using the polyol process.

\section{High temperature and hydrothermal reactions}

Dispersible magnetic iron oxide NPs can be obtained by the decomposition of $\mathrm{Fe}$ precursors in the proximity of hot organic surfactants. Hydrothermal synthesis of $\mathrm{Fe} 3 \mathrm{O} 4$ is realized in aqueous media, in reactors with sustain high pressures and temperatures. There are two reactions that can result in the formation of ferrites: hydrolysis and oxidation of mixed metal hydroxides. The only difference between the two is that in hydrolysis, ferrous salts are used. The main characteristics that have an impact on the final product are temperature, time and the solvent. If during the reaction time is longer, the size range of the $\mathrm{Fe} 3 \mathrm{O} 4$ powders is higher. Using thermal decomposition, hydrophobic magnetite particles were synthesized from Fe (CO) 5 in an octylether solution of oleic acid. Sol-gel reactions the solgel process uses molecular precursors that go through hydroxylation and condensation in a solution. The reactions are conducted at room temperature, thus additional heat treatments is needed in order to achieve the final crystalline shape. Solvent, temperature, concentration and $\mathrm{pH}$ are some of the parameters that influence in properties of the gel. This method can attain strict control over the particle size, monodispersity and predetermined structure. An example is the synthesis of maghemite NPs with a size range between 6 $\mathrm{nm}$ and $15 \mathrm{~nm}$, after a heat treatment of the gels at high temperature $(4000 \mathrm{C})^{78}$.

\section{Aerosol/vapor methods}

Precise shaped and sized magnetic nanoparticles can be obtained using spray and laser pyrolysis. These methods have a high manufacturer rate that foresees a promising future for the synthesis of magnetic NPs used in vivo and in vitro applications. In spray pyrolysis process, a solid is obtained by spraying a solution in a succession of reactors. In these reactors, the aerosol droplets are subjected to evaporation of the solvent and solute condensation in the droplet. The following step is drying and thermolysis of the precipitation particle at higher temperatures. Finally, the results are microporous solids, from which in the end dense particles are formed. The shape and size of the final particles can be managed from the original droplets. Laser pyrolysis consists in heating a flowing mixture of gases with a continuous wave carbon dioxide laser. The laser initiates and maintains a chemical reaction. This method can manufacture small sized particles, with narrow particle size distribution and also an almost absence of aggregation. By using a one-step $\mathrm{CO} 2$ laser pyrolysis

\section{Sonolysis}

Iron oxide can be synthesized by sonolysis of organometallic precursors. High temperature is generated by the fast collapse of the cavities (cavitation process) and ferrous salts are conversed into magnetite NPs.

\section{pH sensitive core-shell magnetic nanoparticles}

In the last years, there had been an intense interest in the manufacturing of core-shell magnetic NPs. Core-shell magnetic nanoparticles can merge the magnetic properties given by magnetic NPs as the core and the properties of the shell, depending on the material ${ }^{79}$. Core-shell NPs have unique combined properties such as lower cytotoxicity, higher dispensability, biocompatibility and chemical and thermal stability. Occasionally, the shell does not only have the property of increasing biocompatibility, but also of improving the cores properties. The hydrophobicity of nanoparticles is an important aspect when the NPs are dispersed in aqueous media. Core shell nanoparticles have been intensively investigated in the field of cancer therapy. The core is coated with a convenient shell in the concern of increasing biocompatibility and pharmacokinetic properties in the human body. The anticancer agent is either attached or encapsulated in/on the core-shell complex. Higher surface leads to higher drug storing capacity. Surface modification must be made in respect of releasing the anticancer agent in the tumor site by means of changes in the $\mathrm{pH}$ values ${ }^{80}$. The $\mathrm{pH}$ value of damaged tissues, when affected by inflammation, infection or cancer is substantially different than the $\mathrm{pH}$ of healthy tissues. For example, if the tissue is subjected to a 60-hour inflammatory reaction, its $\mathrm{pH}$ value drops from 7.4 to 6.5 . Specific targeting of pharmaceutical drugs in the tumor site 
is highly important in cancer therapy ${ }^{81}$. In core-shell nanoparticles with directly connected functionalities, the shell must be made of organic or inorganic materials that are directly grown or connected to the magnetic core in order to achieve another performance apart from their magnetic one. Magnetic NPs have been widely used in biomedical applications that require high biocompatibility; therefore, surface functionalization is an essential requirement in the interest of making them applicable in this field. Multi functionalized nanocomposites consist of a chemotherapeutic agent (discharged by a $\mathrm{pH}$ sensitive mechanism), a specific ligand that can relate to tumor cells and iron oxide NPs as a core ${ }^{82}$. Even though iron oxides have many crystalline polymorphs, only magnetite (Fe3O4) and maghemite $(\gamma$-Fe2O3 $)$ are used in biomedical applications. Their surface chemistry and functionalization can also be easily fashioned. In order to improve drug delivery, adequate surface coating must be realized to become an integral component of a multifunctional magnetic NPs platform. To achieve this, an iron oxide core can be coated with organic materials (e.g., PEG), inorganic materials (e.g., gold) or oxides (e.g., silica) ${ }^{83}$. The magnetic polymer-coated NPs, loaded with specific tumor drug is guided to the desired location by means of an external magnetic field. When the magnetic field is removed, the nanovesicles will detect the cancerous cells by tumor recognition function and the drug will be released because of the change in $\mathrm{pH}$ (from physiological $\mathrm{pH}-7.4$ to endosomal $\mathrm{pH}-5.3$ ). The drug release can be accelerated by a magnetic field ${ }^{84}$. $\mathrm{pH}$ responsive polymers can modify their size in function of the changes in the $\mathrm{pH}$ of the surrounding environment, resulting in a expansion or a collapse, depending on the $\mathrm{pH}$ of the media. This behavior is due to the existence of specific functional groups in the polymer chains. The $\mathrm{pH}$ sensitive materials can be classified in two groups. In the first one there are polymers containing acidic groups, such as $-\mathrm{COOH}$, which expand in basic $\mathrm{pH}$ (e.g., polyacrylic acid). The second one consists of polymers with basic groups, as $-\mathrm{NH} 2$, which swell in acidic $\mathrm{pH}$ (case of chitosan). $\mathrm{pH}$ dependent expansion of nanogels can also be used for the drugs loading. When the $\mathrm{pH}-$ sensitive nanosized gel swells, the permeability increases, thus allowing the encapsulation of NPs or the release of an already incorporated drug ${ }^{85}$.

\section{Applications in cancer therapy}

Cancer is known to be the leading cause of mortality worldwide. This cause is determined by the rapid and uncontrolled cell growth. The most used treatment to kill cancerous cells is chemotherapy. However, chemotherapy treatment comes with severe sides effects, because of its lack of specificity, affecting also healthy cells. In order to overcome the downsides of chemotherapy, an effective approach is the use of systemic nanomedicine with anticancer drugs. Presently, multifunctional nanoparticles platforms have been used as target drug delivery systems.

Magnetic nanoparticles coated with polymeric materials have higher stability, superparamagnetism and suitable biocompatibility as drug vesicles ${ }^{86}$.
Doxorubicin (DOX) was used as an anticancer drug. The anticancer drug attached was DOX. It was shown that PAA and FA shell could encapsulate a big amount of DOX and showed remarkable efficiency for targeting [87].

Nanomaterials are intense researched because of their unique properties that make them promising for biomedical applications. Magnetic nanoparticles have super paramagnetic properties, meaning that when the magnetic field is removed, they can redisperse. Core-shell magnetite hybrid NPs have the advantage of high stability against oxidation and also, because of the polymeric shell, a great amount of drug can be encapsulated. Poly-2-dimethyl amino ethyl methacrylate (PDMAEMA) has excellent properties, such as biocompatibility, hydrophilicity and $\mathrm{pH}$ responsivity, which make them suitable for medical and pharmaceutical studies. ${ }^{88}$

The silica shell preserves the magnetism of Fe3O4. However, Fe3O4@SiO2 cannot load drugs properly, so the need of a more complex system is desired. Multifunctional hybrid NPs were obtained by attaching to the silica coating the biocompatible block polymer polyethylene glycolblock-polyaspartic acid (PEG-b-PAsp). This enabled the stabilization of the magnetic NPs and also avoided absorption. PAsp was used in order to encapsulate the therapeutic drug (DOX) via electrostatic interaction. This kind of hybrid NPs showed great potential applications in cancer therapy as targeting drug delivery carriers ${ }^{89}$.

Nanoparticles smaller than $200 \mathrm{~nm}$ will avoid the RES recognition when coated with PEG, a hydrophilic and biocompatible polymer. An example of such NPs is the PEG-b-poly-D, L-lactide, which contain encapsulated DOX and a superparamagnetic NPs cluster. The used cytostatic is adriamycin (ADR), encapsulated into the PGMA-coated core by simply blending them in an aqueous solution at physiological $\mathrm{pH}$. When reaching a $\mathrm{pH}$ lower than 5.5, the drug was released. This formula showed low cytotoxicity because of the MPEG shell ${ }^{90}$.

When the $\mathrm{pH}$ value was 7.4 , less than $35 \%$ of the drug was released in 50 hours; when the $\mathrm{pH}$ value dropped to 5.5, $80 \%$ of the drug was released in the same amount of time. To improve targeted drug delivery in cancer therapy, a system consisting of titanium oxide encapsulated iron oxide NPs (Fe3O4@TiO2) have been developed. An anticancer drug, DOX, was loaded in the core-shell structure. Fe3O4@TiO2 nanoparticles display pH sensitive release of DOX in the desired area. These NPs could be used in the treatment of cancer with chemo-sonodynamic therapy ${ }^{91-92}$.

\section{CONCLUSIONS}

The state-of-the-art of $\mathrm{pH}$-responsive drug delivery using mesoporous nanoparticles has been deeply revised. These stimuli-responsive nanocarriers are based on the small variations in $\mathrm{pH}$ between healthy and diseased areas along the body. Those variations are used to trigger the release of the cargo through different mechanisms. We have considered three different approximations of $\mathrm{pH}$-responsive nanocarriers. The host-guest interactions have been shown to be useful to retain the drugs at physiological $\mathrm{pH}$. However, it would be desirable to minimize the amount of 
drugs released outside the target area despite the electrostatic interactions. Regarding the $\mathrm{pH}$-responsive nanocarriers, it has been shown in this review that it is possible to design hybrid nanoparticles capable of triggering the release in very specific situations through the subtle changes in $\mathrm{pH}$. However, it is still difficult for all of the nanocarriers injected to reach the diseased area. Then, future work should be directed toward synthesizing smart $\mathrm{pH}$-responsive nanocarriers with enhanced targeting ability, capable of avoiding the different barriers present in the body and accumulating only in the diseased tissues to increase the efficacy of the therapy.Nevertheless, recent

\section{REFERENCES:}

1. Nogueira E, Gomes AC, Preto A, Cavaco-Paulo, A.Folate-targeted nanoparticles for rheumatoid arthritis therapy. Nano med. Nanotechnol. Biol. Med. $2015 ;(12) 1113-1126$

2. Brede C, Labhasetwar V. Applications of nanoparticles in the detection and treatment of kidney diseases. Adv. Chronic Kidney Dis. 2013; (20):454-465.

3. Azarmi S Roa, WH Löbenberg, R Targeted delivery of nanoparticles for the treatment of lung diseases. Adv. Drug Deliv. Rev. 2008; (60)863875.

4. Sun L, Zhang X, Wu Z, Zheng C, Li C. Oral glucose- and pH-sensitive nanocarriers for simulating insulin release in vivo. Polym. Chem. 2014; (5):1999-2009.

5. Spuch, C, Saida O, Navarro C. Advances in the treatment of neurodegenerative disorders employing nanoparticles. Recent Pat. Drug Deliv. Formul.2012; (6):2-18.

6. Schlapschy ,Binder U, Börger C, Theobald I,Wachinger K, Kisling S, Haller D, Skerra A. PA Sylation: A biological alternative to PEGylation for extending the plasma half-life of pharmaceutically active proteins. Protein Eng. Des. Sel. 2013; (26):489-501.

7. Estephan ZG, Schlenoff PS, Schlenoff JB Z witteration as an alternative to PEGylation. Langmuir 2011; (27):6794-6800.

8. Joker's JV, Lobovkina T, Zane RN, Gambhir SS. Nanoparticle PEGylation for imaging and therapy. Nanomedicine (Lond.) 2011; (6):715-728

9. Fang J, Nakamura $\mathrm{H}$, Maeda $\mathrm{H}$. The EPR effect: Unique features of tumor blood vessels for drug delivery, factors involved, and limitations and augmentation of the effect. Adv. Drug Deliv. Rev. 2011 ;(63):136151.

10. Yameen, B.; Choi, W.I.; Vilos, C.; Swami, A.; Shi, J.; Farokhzad, O.C. Insight into nanoparticle cellular uptake and intracellular targeting. J. Control. Release 2014 (190), 485-499.

11. Wang A, Langer RS, Farokhzad, O. Nanoparticle Delivery of Cancer Drugs. Annu. Rev. Med. 2011; (63), 85-98.

12. Duncan R, Gaspar R. Nanomedicine(s) under the microscope. Mol. Pharm. 2011; (8): 2101-2141.

13. Chen Y, Shi J. Mesoporous carbon biomaterials. Sci. China Mater. 2015; (58):241-257.

14. Ugazio E, Gastaldi L, Brielle V, Scalarone D, Jadhav SA, Oliaro-bosso S, Zonari D, Berlier G, Miletto I, Sapino S. Thermoresponsive mesoporous silica nanoparticles as a carrier for skin delivery of quercetin. Int. J. Pharm. 2016; (511):446-454

15. Jadhav SA, Miletto I, Brunella V, Berlier G, Scalarone D. Controlled post-synthesis grafting of thermoresponsive poly (Nisopropylacrylamide) on mesoporous silica nanoparticles. Polym. Adv. Technol. 2015; (26):1070-1075.

16. Kim J, Kim HS, Lee N, Kim T, Kim H, Yu T, Song IC, Moon WK, Hyeon T. Multifunctional uniform nanoparticles composed of a magnetite nanocrystal core and a mesoporous silica shell for magnetic resonance and fluorescence imaging and for drug delivery. Angew. Chem. Int. Ed. 2008; (47):8438-8441.

17. An J, Zhang X, Guo, Q.; Zhao, Y.; Wu, Z.; Li, C. Glycopolymer modified magnetic mesoporous silica nanoparticles for MR imaging and targeted drug delivery. Colloids Surf. A Physicochem. Eng. Asp. 2015; (482):98-108.

18. Wang D, Wu S. Red-Light-Responsive Supramolecular Valves for Photocontrolled Drug Release from Mesoporous Nanoparticles. Langmuir 2016; (32):632-636. advances in the polymer design, structure-property correlation, mechanistic understanding, and applications of a multitude of $\mathrm{pH}$-sensitive nanomaterials reviewed here provide general guidelines for future rational design of more effective $\mathrm{pH}$-responsive nanomaterials for cancer diagnosis and treatment.

\section{ACKNOWLEDGEMENT}

Authors are sincerely thankful to Anuradha College of Pharmacy, Chikhli Dist- Buldana M.S. India. For providing necessary facilities for work and authors also thankful to lovely friends.

19. He D He X Wang K, Cao J, Zhao Y. A light-responsive reversible molecule-gated system using thymine-modified mesoporous silica nanoparticles. Langmuir 2012; (28):4003-4008.

20. Martínez-Carmona, M, Baeza A, Rodriguez-Milla MA, García-Castro J, Vallet-Regí M. Mesoporous silica nanoparticles grafted with a lightresponsive protein shell for highly cytotoxic antitumoral therapy. J. Mater. Chem. B 2015; (3):5746-5752.

21. Paris JL, Cabañas MV, Manzano M, Vallet-Regí M. Polymer-Grafted Mesoporous Silica Nanoparticles as Ultrasound-Responsive Drug Carriers. ACS Nano 2015; (9):11023-11033.

22. Guisasola E, Baeza A, Talelli M, Arcos D, Vallet-Regí M. Design of thermoresponsive polymeric gates with opposite controlled release behaviors. RSC Adv. 2016; 6:42510-42516.

23. Guisasola E, Baeza A, Talelli M, Arcos D, Moros M, De La Fuente JM, Vallet-Regí M. Magnetic-Responsive Release Controlled by Hot Spot Effect. Langmuir 2015; (31): 12777-12782.

24. Gondi CS, Rao, J.S. Cathepsin B as a cancer target. Expert Opin. Ther. Targets 2013; (17): 281-291.

25. Huo, M.; Yuan, J.; Tao, L.; Wei, Y. Redox-responsive polymers for drug delivery: From molecular design to applications. Polym. Chem. 2014; (5):1519.

26. Liu, J.; Zhang, B.; Luo, Z.; Ding, X.; Li, J.; Dai, L.; Zhou, J.; Zhao, X.; Ye, J.; Cai, K. Enzyme responsive mesoporous silica nanoparticles for targeted tumor therapy in vitroandin vivo. Nanoscale2015; (7):36143626

27. Liu, Y.; Ding, X.; Li, J.; Luo, Z.; Hu, Y.; Liu, J.; Dai, L.; Zhou, J.; Hou, C.; Cai, K. Enzyme responsive drug delivery system based on mesoporous silica nanoparticles for tumor therapy in vivo. Nanotechnology 2015; (26):145102.

28. Li, Z.-Y.; Hu, J.-J.; Xu, Q.; Chen, S.; Jia, H.-Z.; Sun, Y.-X.; Zhuo, R.X.; Zhang, X.-Z. A redox-responsive drug delivery system based on RGD containing peptide-capped mesoporous silica nanoparticles. J.Mater. Chem.B 2015; (3):39-44.

29. Zhao,Q.;Geng,H.;Wang,Y.;Gao,Y.;Huang,J.;Wang,Y.;Zhang,J.;Wang,S .Hyaluronicacidoligosaccharide modified redox-responsive mesoporous silica nanoparticles for targeted drug delivery. ACS Appl. Mater. Interfaces 2014; (6):20290-20299.

30. Lee, C.-H.; Lo, L.-W.; Mou, C.-Y.; Yang, C.-Synthesis and characterization of positive-charge functionalized mesoporous silica nanoparticles for oral drug delivery of an anti-inflammatory drug. Adv. Funct. Mater. 2008; (18):3283-3292.

31. Shao,D.;Zhang,X.;Liu,W.;Zhang,F.;Zheng,X.;Qiao,P.;Li,J.;Dong,W.;Ch en,L.JanusSilver-Mesoporous Silica Nanocarriers for SERS Traceable and $\mathrm{pH}$-Sensitive Drug Delivery in Cancer Therapy. ACS Appl. Mater. Interfaces 2016; (8):4303-4308

32. Rasouli, S.; Davaran, S.; Rasouli, F.; Mahkam, M.; Salehi, R. Positively charged functionalized silica nanoparticles as nontoxic carriers for triggered anticancer drug release. Des. Monomers Polym. 2014; (17):227-237.

33. Tacar, O.; Sriamornsak, P.; Dass, C.R. Doxorubicin: An update on anticancer molecular action, toxicity and novel drug delivery systems. J. Pharm. Pharmacol. 2013; (65):157-170.

34. Zheng, H.; Tai, C.-W.; Su, J.; Zou, X.; GAO, F. Ultra-small mesoporous silica nanoparticles as efficient carriers for $\mathrm{pH}$ responsive releases of anti-cancer drugs. Dalton Trans. 2015; (44):20186-20192.

35. Moorthy, M.S.; Bae, J.H.; Kim, M.J.; Kim, S.H.; Ha, C.S. Design of a novel mesoporous organosilica hybrid microcarrier: A $\mathrm{pH}$ stimuliresponsive dual-drug-delivery vehicle for intracellular delivery of anticancer agents. Part. Part. Syst. Charact. 2013; (30):1044-1055.

36. Bae, Y.; Fukushima, S.; Harada, A.; Kataoka, K.J.A.C. Design of environment-sensitive supramolecular assemblies for intracellular drug 
delivery: Polymeric micelles that are responsive to intracellular $\mathrm{pH}$ change. Angew. Chem.-Int. Edit. 2003; (115):4788-4791.

37. Dai, S.; Ravi, P.; Tam, K.C.pH-Responsive polymers: Synthesis, properties and applications. Soft Matter 2008; (4):435-449.

38. Tang, H.; Luan, Y.; Yang, L.; Sun, H. A Perspective on Reversibility in Controlled Polymerization Systems: Recent Progress and New Opportunities. Molecules 2018; (23): 2870.

39. Sun, H.; Kabb, C.P.; Dai, Y.; Hill, M.R.; Ghiviriga, I.; Bapat, A.P.; Sumerlin, B.S. Macromolecular metamorphosis via stimulus-induced transformations of polymer architecture. Nat. Chem. 2017; (9):817-823.

40. Sun, H.; Kabb, C.P.; Sumerlin, B.S. Thermally-labile segmented hyperbranched copolymers: Using reversible-covalent chemistry to investigate the mechanism of self-condensing vinyl copolymerization. Chem. Sci. 2014; (5):4646-4655.

41. Sun,H.; Kabb,C.P.; Sims,M.B.; Sumerlin, B.S.Architecture-transform able polymers: Reshaping the future of stimuli-responsive polymers. Prog. Polym. Sci. 2018, in press.

42. Kocak, G.; Tuncer, C.; Bütün, V. pH-Responsive polymers. Polym. Chem. 2017; (8):144-176.

43. Bazban-Shotorbani,S.; Hasani-Sadrabadi, M.M.;Karkhaneh,A.; Serpooshan,V.; Jacob, K.I.;Moshaverinia,A.; Mahmoudi, M. Revisiting structure-property relationship of $\mathrm{pH}$-responsive polymers for drug delivery applications. J. Control. Release. 2017; (253):46-63.

44. Ranneh, A.H.; Takemoto, H.; Sakuma, S.; Awaad, A.; Nomoto, T.; Mochida, Y.; Matsui, M.; Tomoda, K.; Naito, M.; Nishiyama, N. An Ethylenediamine-based Switch to Render the Polyzwitterion Cationic at Tumorous $\mathrm{pH}$ for Effective Tumor Accumulation of Coated Nanomaterials. Angew. Chem. Int. Ed. 2018; (57):5057-5061.

45. Mizuhara, T.; Saha, K.; Moyano, D.F.; Kim, C.S.; Yan, B.; Kim, Y.K.; Rotello, V.M. Acylsulfonamide-Functionalized Zwitterionic Gold Nanoparticles for Enhanced Cellular Uptake at Tumor pH. Angew. Chem. Int. Ed. 2015; (54):6567-6570.

46. Li, Y.; Wang, Z.; Wei, Q.; Luo, M.; Huang, G.; Sumer, B.D.; Gao, J. Non-covalent interactions in controlling $\mathrm{pH}$-responsive behaviors of self-assembled nanosystems. Polym. Chem. 2016; (7):5949-5956.

47. Ma, X.; Wang, Y.; Zhao, T.; Li, Y.; Su, L.-C.; Wang, Z.; Huang, G.; Sumer, B.D.; Gao, J. Ultra-pH-Sensitive Nanoprobe Library with Broad pH Tunability and Fluorescence Emissions. J. Am. Chem. Soc. 2014; (136):11085-11092.

48. Li, Y.; Wang, Y.; Huang, G.; Gao, J. Cooperativity Principles in SelfAssembled Nanomedicine. Chem. Rev. 2018; (118):5359-5391

49. Li, Y.; Wang, Y.; Huang, G.; Ma, X.; Zhou, K.; Gao, J. ChaotropicAnion-Induced Supramolecular Self-Assembly of Ionic Polymeric Micelles. Angew. Chem. Int. Edit. 2014; (53):8074-8078.

50. Wang, C.; Zhao, T.; Li, Y.; Huang, G.; White, M.A.; Gao, J. Investigation of endosome and lysosome biology by ultra $\mathrm{pH}$-sensitive nanoprobes. Adv. Drug Deliv. Rev. 2017; (113):87-96.

51. Fu, L.; Yuan, P.; Ruan, Z.; Liu, L.; Li, T.; Yan, L. Ultra-pH-sensitive polypeptide micelles with large fluorescence off/on ratio in near infrared range. Polym. Chem. 2017; (8):1028-1038.

52. Kolb, H.C.; Finn, M.G.; Sharpless, K.B. Click Chemistry: Diverse Chemical Function from a Few Good Reactions. Angew. Chem. Int. Edit. 2001; (40):2004-2021.

53. Weerakkody, D.; Moshnikova, A.; Thakur, M.S.; Moshnikova, V.; Daniels, J.; Engelman, D.M.; Andreev, O.A.; Reshetnyak, Y.K. Family of $\mathrm{pH}$ (low) insertion peptides for tumor targeting. P. Natl. Acad. Sci. USA. 2013; (110):5834-5839.

54. Segala, J.; Engelman, D.M.; Reshetnyak, Y.K.; Andreev, O.A. Accurate analysis of tumor margins using a fluorescent $\mathrm{pH}$ low insertion peptide (pHLIP). Int. J. Mol. Sci. 2009 (10), 3478-3487.

55. Barrera, F.N.; Fendos, J.; Engelman, D.M. Membrane physical properties influence transmembrane helix formation. P. Natl. Acad. Sci. USA. 2012: (109):14422-14427.

56. Nguyen, V.P.; Alves, D.S.; Scott, H.L.; Davis, F.L.; Barrera, F.N. A novel soluble peptide with $\mathrm{pH}$-responsive membrane insertion. Biochemistry. 2015; (54):6567-6575.

57. Emmetiere,F.; Irwin,C.; Viola-Villegas,N.T.; Longo,V.; Cheal,S.M.; Zanzonico,P.; Pillarsetty,N.; Weber,W.A.; Lewis, J.S.; Reiner, T. 18Flabeled-bioorthogonal liposomes for in vivo targeting. Bioconjugate Chem. 2013; (24):1784-1789.

58. Wyatt, L.C.; Lewis, J.S.; Andreev, O.A.; Reshetnyak, Y.K.; Engelman, D.M. Applications of pHLIP technology for cancer imaging and therapy. Trends Biotechnol. 2017; (35):653-664.

59. Hunt, J.F.; Rath, P.; Rothschild, K.J.; Engelman, D.M. Spontaneous, $\mathrm{pH}$-dependent membrane insertion of a transbilayer $\alpha$-helix. Biochemistry 1997; (36):15177-15192.
60. Reshetnyak, Y.K.; Andreev, O.A.; Lehnert, U.; Engelman, D.M. Translocation of molecules into cells by pH-dependent insertion of a transmembrane helix. P. Natl. Acad. Sci. USA. 2006; (103):6460-6465.

61. Musial-Siwek, M.; Karabadzhak, A.; Andreev, O.A.; Reshetnyak, Y.K.; Engelman, D.M. Tuning the insertion properties of pHLIP. BBABiomembranes. 2010; (1798):1041-1046.

62. Platt,V.M.;SzokaJr,F.C Anticancer therapeutics: Targeting macromolecules and nanocarriers to hyaluronan or CD44, a hyaluronan receptor. Mol. Pharm. 2008; (5):474-486.

63. Miyazaki, M.; Yuba, E.; Hayashi, H.; Harada, A.; Kono, K. Hyaluronic acid-based $\mathrm{pH}$-sensitive polymer-modified liposomes for cell-specific intracellular drug delivery systems. Bioconjugate Chem. 2017; (29):4455.

64. Wei, H.; Zhuo, R.; Zhang, X. Design and development of polymeric micelles with cleavable links for intracellular drug delivery. Prog. Polym. Sci. 2013; (38):503-535.

65. Zhang, X.; Malhotra, S.; Molina, M.; Haag, R. Micro- and nanogels with labile crosslinks - from synthesis to biomedical applications. Chem. Soc. Rev. 2015; (44):1948-1973.

66. Tang, H.; Tsarevsky, N.V. Lipoates as building blocks of sulfurcontaining branched macromolecules. Polym. Chem. 2015; (6):69366945.

67. Suvarapu, L.N.; Seo, Y.K.; Baek, S.-O.; Ammireddy, V.R. Review on analytical and biological applications of hydrazones and their metal complexes. E-J Chem. 2012; (9): 1288-1304.

68. Lee, K.Y.; Wang, Y.; Nie, S. In vitro study of a pH-sensitive multifunctional doxorubicin-gold nanoparticle system: Therapeutic effect and surface enhanced Raman scattering. RSC Adv. 2015; (5):65651-65659.

69. Hu, J.; Xie, L.; Zhao, W.; Sun, M.; Liu, X, Gao W. Design of tumorhoming and $\mathrm{pH}$-responsive polypeptide-doxorubicin nanoparticles with enhanced anticancer efficacy and reduced side effects. Chem Commun. 2015; (51):11405-11408.

70. Huang, L.; Tao, K.; Liu, J.; Qi, C.; Xu, L.; Chang, P.; Gao, J.; Shuai, X.; Wang, G.; Wang, Z. interfaces, Design and fabrication of multifunctional sericin nanoparticles for tumor targeting and $\mathrm{pH}$ responsive subcellular delivery of cancer chemotherapy drugs. ACS Appl. Mater. Inter. 2016; (8):6577-6585.

71. Liu, B.; Thayumanavan, S. Substituent effects on the $\mathrm{pH}$ sensitivity of acetals and ketals and their correlation with encapsulation stability in polymeric nanogels. J. Am. Chem. Soc. 2017; (139):2306-2317.

72. Chen,Y.;Ai,K.;Liu,J.;Sun,G.;Yin,Q.;Lu,L.Multifunctional envelope-type mesoporous silica nanoparticles for $\mathrm{pH}$-responsive drug delivery and magnetic resonance imaging. Biomaterials 2015; (60):111-120.

73. Wang, L.; Liu, G.; Wang, X.; Hu, J.; Zhang, G.; Liu, S. Aciddisintegratable polymersomes of $\mathrm{pH}$-responsive amphiphilic diblock copolymers for intracellular drug delivery. Macromolecules 2015; (48):7262-7272.

74. Huang,F.;Cheng,R.;Meng,F.;DEng, C.; Zhong, Z.Micelles based on acid degradablepoly (acetalurethane): Preparation, $\mathrm{pH}$-sensitivity, and triggered intracellular drug release. Biomacromolecules 2015; (16):2228-2236.

75. Cao H, Chen C, Xie D, Chen X, Wang P, Wang Y, Song H, Wang W. A hyperbranched amphiphilic acetal polymer for $\mathrm{pH}$-sensitive drug delivery. Polym. Chem. 2018; (9):169-177.

76. Louage B, Zhang Q, Vanparijs N, Voorhaar L, Vande Casteele S, Shi Y, Hennink WE, Van Bocxlaer J, Hoogenboom R, De Geest, B.G. Degradable Ketal-Based Block Copolymer Nanoparticles for Anticancer Drug Delivery: A Systematic Evaluation. Biomacromolecules. 2015; (16):336-350.

77. Tartaj P, del Puerto Morales M, Veintemillas-Verdaguer S, GonzálezCarreño T, Serna CJ. The preparation of magnetic nanoparticles for applications in biomedicine. J Phys D Appl Phys. 2003; 36(13):R182.

78. Laurent S, Forge D, Port M, Roch A, Robic C, Vander Elst L, Muller RN. Magnetic iron oxide nanoparticles: synthesis, stabilization, vectorization, physicochemical characterizations, and biological applications. Chem Rev, 2008; 108(6):2064-2110.

79. Kalska-Szostko B, Wykowska U, Satuła D. Magnetic nanoparticles of core-shell structure. Colloids Surf A Physicochem Eng Asp, 2015; 481:527-536.

80. Chatterjee K, Sarkar S, Jagajjanani Rao K, Paria S. Core/shell nanoparticles in biomedical applications. Adv Colloid Interface Sci. 2014; 209:8-39.

81. Ganta S, Devalapally H, Shahiwala A, Amiji M. A review of stimuliresponsive nanocarriers for drug and gene delivery. J Control Release, 2008; 126(3):187-204. 
82. Salgueiriño-Maceira V, Correa-Duarte MA. Increasing the complexity of magnetic core/shell structured nanocomposites for biological applications. Adv Mater, 2007; 19(23):4131-4144.

83. Misra RDK. Core-shell magnetic nanoparticle carrier for targeted drug delivery: challenges and design. Mater Technol Adv Performance Mater; 2010; 25(3-4):118-126.

84. Yan H, Ga M, Liu K. Chapter 12: Multifunctional magnetic hybrid nanoparticles as a nanomedical platform for cancertargeted imaging and therapy. In: Ghista DN (ed). Biomedical science, engineering and technology, InTech, Rijeka, Croatia. 2012; 283-300.

85. Medeiros SF, Santos AM, Fessi H, Elaissari A. Stimuliresponsive magnetic particles for biomedical applications. Int J Pharm. 2011; 403(1-2):139-161.

86. Yang C, Guo W, Cui L, An N, Zhang T, Lin H, Qu F. pH responsive magnetic core-shell nanocomposites for drug delivery. Langmuir. 2014; 30(32):9819-9827.

87. Shen YQ, Pu ML, Jiang W. Polymer-tethered $\mathrm{pH}$-sensitive magnetic nanoparticles for targeted delivery of anti-cancer drug. Adv Mater Res. 2014; 936:723-727.
88. Zhou L, Yuan J, Yuan W, Sui X, Wu S, Li Z, Shen D. Synthesis, characterization, and controllable drug release of $\mathrm{pH}$-sensitive hybrid magnetic nanoparticles. J Magn Mater, 2009. 321(18):2799-2804.

89. Wu CL, He H, Gao HJ, Liu G, Ma RJ, An YL, Shi LQ. Synthesis of Fe3O4@SiO2@polymer nanoparticles for controlled drug release. Sci China Chem. 2010; 53(3):514-518.

90. Guo M, Yan Y, Zhang H, Yan H, Cao Y, Liu K, Wan S, Huang J, Yue $\mathrm{W}$. Magnetic and $\mathrm{pH}$-responsive nanocarriers with multilayer core-shell architecture for anticancer drug delivery. J Mater Chem. 2008; 18(42):5104-5112.

91. Yu S, Wu G, Gu X, Wang J, Wang Y, Gao H, et al. Magnetic and $\mathrm{pH}-$ sensitive nanoparticles for antitumor drug delivery. Colloids Surf B Biointerfaces. 2013; 103:15-22.

92. Shen S, Wu L, Liu J, Xie M, Shen H, Qi X, Yan Y, Ge Y, Jin Y. Coreshell structured Fe3O4@TiO2-doxorubicin nanoparticles for targeted chemo-sonodynamic therapy of cancer. Int J Pharm, 2015; 486(12):380-388. 